\title{
Thermo-Electro-Mechanics at Individual Particles in \\ Complex Colloidal Systems
}

\section{Supplementary Information}

Pavana Siddhartha Kollipara ${ }^{1}$ Linhan Lin ${ }^{1,2,3, *}$, and Yuebing Zheng ${ }^{1,2, *}$

${ }^{1}$ Walker Department of Mechanical Engineering, The University of Texas at Austin, Austin, TX, 78712, USA.

${ }^{2}$ Materials Science \& Engineering Program and Texas Materials Institute, The University of Texas at Austin, Austin, TX, 78712, USA.

${ }^{3}$ Department of Precision Instruments, Tsinghua University, Beijing 100084, People's Republic of China

*E-mail: $\underline{\text { linlh2019@ mail.tsinghua.edu.cn, zheng@austin.utexas.edu }}$ 


\section{Supplementary Notes}

\section{Supplementary Note I - Soret coefficient of micelles}

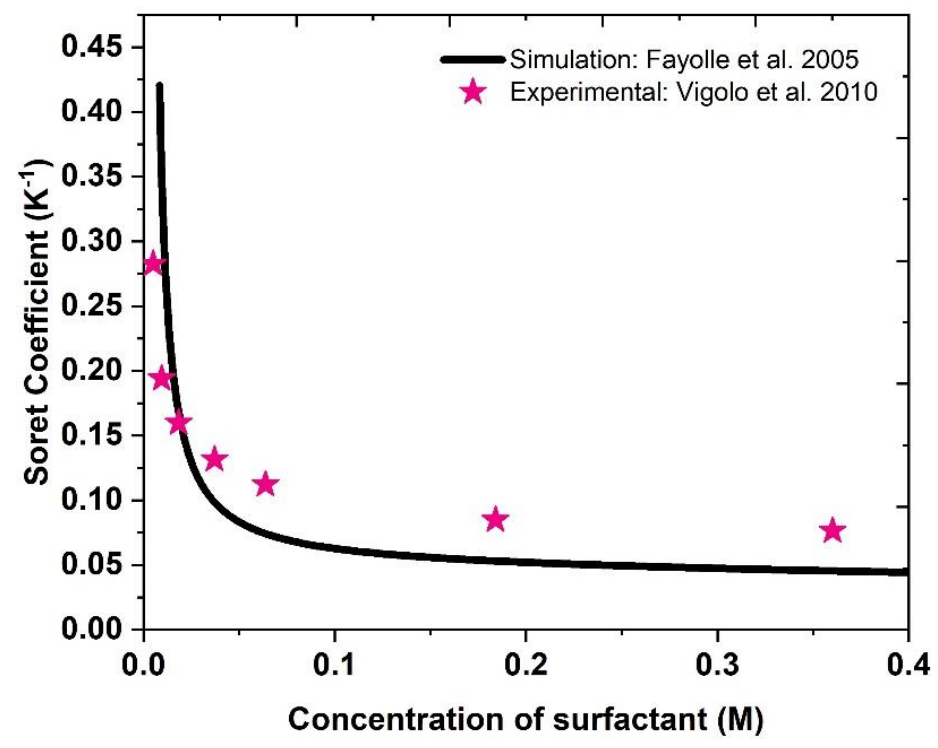

Figure S1: Soret Coefficient of SDS vs concentration of surfactant: B lack line indicates the simulated values ${ }^{1}$ and red stars indicate experimental values ${ }^{2}$.

Soret coefficient of micelles $\left(S_{T, \text { micelle }}\right)$ is retrieved from the work of Fayolle et al. ${ }^{1} . S_{T, \text { micelle }}$ is given as a function of temperature $(T)$, Inverse debye length $(\kappa)$, number density of micelles $(n)$ and the radius of the micelle $\left(r_{\text {mic }}\right)$ as

$$
S_{\text {T,micelle }}=\frac{1}{T} \frac{1}{1+n v}\left(1+\frac{Z^{2} l_{B} \kappa}{4\left(1+\kappa r_{\text {mic }}\right)^{2}}\right)
$$

The virial coefficient $v$ includes the explicit variation of salinity $n_{0}$ as

$$
v=\frac{r_{m i c}(1+2 \chi)}{l_{B} n_{0}}+\frac{\chi(1+\chi)}{\sqrt{32 \pi}\left(l_{B} n_{0}\right)^{\frac{3}{2}}}+4 r_{m i c}^{2} \sqrt{\frac{2 \pi}{l_{B} n_{0}}}
$$

here, $\chi$ is a non-dimensional quantity depending on

$$
\chi=\ln \frac{Z^{2} l_{B}}{2\left(1+\kappa r_{m i c}\right)^{2} r_{m i c}}
$$


and $l_{B}$ is the Bjerrum length and is given as $e^{2} / 4 \pi \epsilon \epsilon_{0} k_{B} T$. Here $\mathrm{Z}$ is the effective charge on the micelle and is a fitting parameter. The radius of micelle is evaluated as ${ }^{3}$

$$
r_{m i c}=0.16+0.1265\left(n_{c}+1\right)+0.0421 n_{c} \mathrm{~nm}
$$

where $n_{c}(=15)$ is the number of carbons in CTAC.

Molecular CTAC would play a significant role in maintaining the salinity of the solution at low surfactant concentration near critical micellar concentration $\left(c_{c m c}\right)$. As surfactant concentration increases, the salinity increases due to increase in micelle concentration, resulting in a decrease in Soret coefficient drastically. At high concentrations of the surfactant, salinity is nearly independent of the molecular CTAC concentration resulting in an asymptotic Soret coefficient. However, depending on micelle parameters like micelle size, dissociation factor, aggregation number etc., the Soret coefficient maintains a complicated relationship with concentration of surfactant and critical micellar concentration which might lead to a different Soret coefficient dependence on the surfactant concentration. To verify the validity of the expression, Figure S1 shows the Soret coefficient of SDS micelles obtained using the parameters of Vigolo et al., ${ }^{2}$ and compared to the experimental values of the Soret coefficient of SDS micelles of the same work. A good agreement is observed between the computed values and the experimentally reported values in the literature 


\section{Supplementary Note II - Derivation of salinity expression}

Salinity is defined as the total concentration of a charged electrolyte assuming that the bulk charge density is zero. The flux equation at steady state is defined as

$$
J_{i}=-D_{i} \nabla c_{i}-c_{i} D_{T, i} \nabla T+\frac{c_{i} Z_{i} e E_{T} D_{i}}{k_{B} T}=0
$$

where $i$ is the index of the particle, $D_{i}$ is diffusion coefficient, $c_{i}$ is the concentration of ions, $D_{T, i}$ is the thermo-diffusion coefficient, $Z_{i}$ is the valency of ions, $E_{T}$ is the thermo-electric field.

$$
\begin{aligned}
& \nabla c_{1}+c_{1} S_{T, 1} \nabla T-\frac{c_{1} Z_{1} e E_{T}}{k_{B} T}=0 \\
& \nabla c_{2}+c_{2} S_{T, 2} \nabla T-\frac{c_{2} Z_{2} e E_{T}}{k_{B} T}=0
\end{aligned}
$$

Using net bulk charge density equation of $Z_{1} c_{1}+Z_{2} c_{2}=0, c_{1}=-Z_{2} c_{2} / Z_{1}$, and using the axiom, 'if $\frac{a_{1}}{a_{2}}=\frac{b_{1}}{b_{2}}$, then $\frac{a_{1}}{a_{2}}=\frac{b_{1}}{b_{2}}=\frac{\left(a_{1}+b_{1}\right)}{\left(a_{2}+b_{2}\right)}$, we have

$$
\frac{\nabla c_{1}}{c_{1}}=\frac{\nabla c_{2}}{c_{2}}=-\left(\frac{Z_{2} S_{T, 1}-Z_{1} S_{T, 2}}{Z_{2}-Z_{1}}\right) \nabla T
$$

For small-gradient approximation, the thermoelectric field created does not separate the ions enough to consider the deviations from salinity. Therefore, the salinity equation would approximately represent the individual normalized concentrations of individual ions. 


\section{Supplementary Note III - Zeta potential meas urements}

Zeta potential for PS particles dispersed in different concentrations of CTAC is measured after sonication for twenty minutes. DLS Zetasizer Nano ZS from Malvern instruments is used to measure the zeta potential. Figure S2 shows the zeta potential as a function of CTAC concentration. Low concentrations of PS particles are utilized to suppress the particle-particle interactions in this work. Therefore, concentrations of CTAC as low as $1 \mathrm{mM}$ was enough for CTAC molecules to adsorb on all PS particles, thereby obtaining a positive zeta potential. The zeta potential at $0 \mathrm{mM} \mathrm{CTAC}$ is negative indicating that the particles are initially negatively charged. As one can observe, there is a steep increasing trend at lower concentration and as concentration increases, zeta potential saturates, indicating a saturation charge on the particle surface, leading a constant trapping force and stiffness.

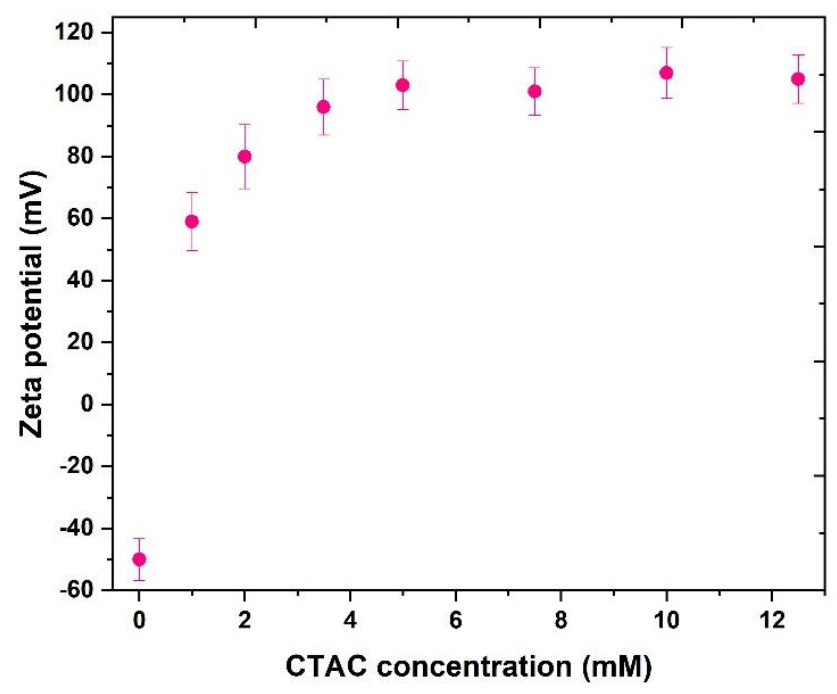

Figure S2: Zeta Potential of charged PS spheres dispersed in CTAC solution as a function of CTAC concentration. Data point at $0 \mathrm{mM} \mathrm{CTAC}$ shows that the PS particles are ne gatively charged without CTAC. 
Supplementary Note IV - Experimental setup, sample preparation and trapping stiffiness determination

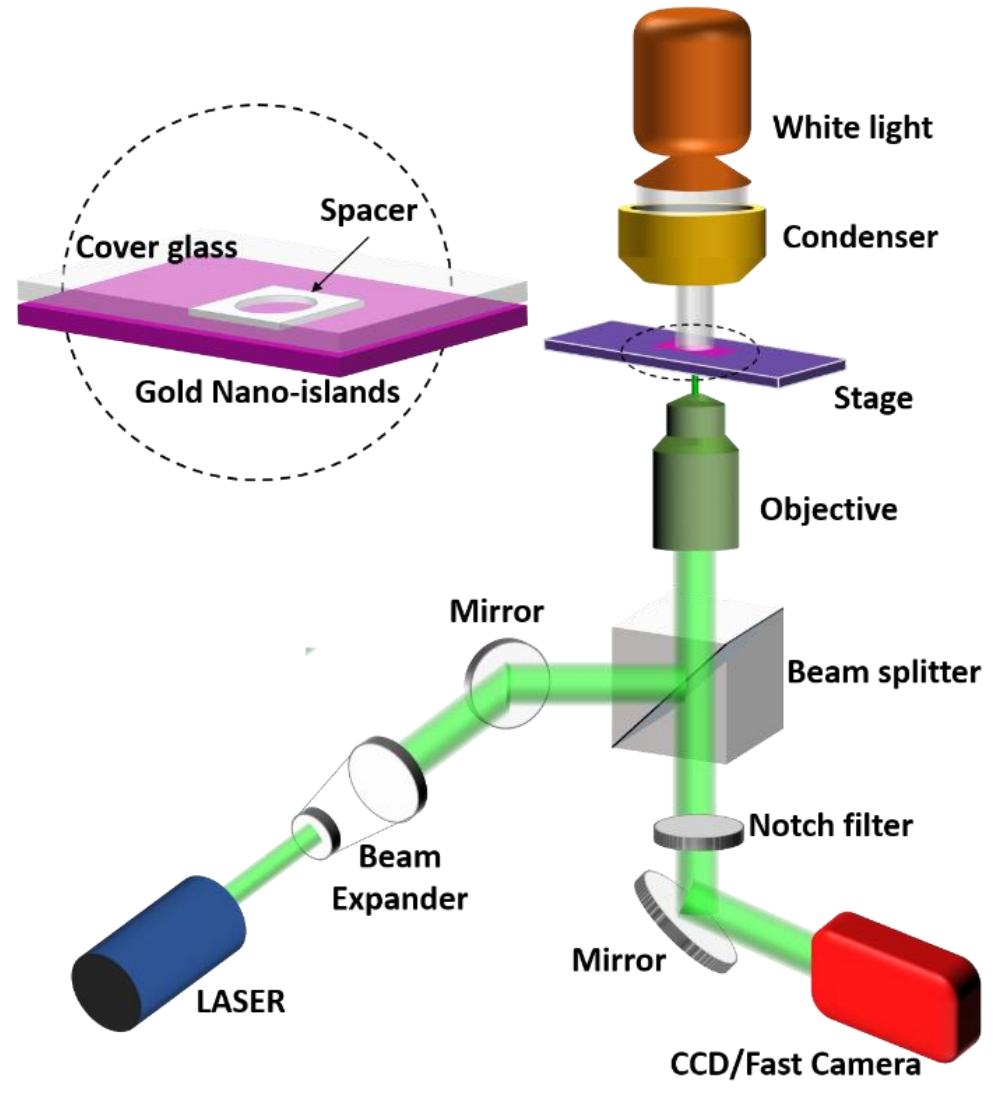

Figure S3: Experimental Setup for OTENT trapping

Figure S3 shows the schematic of experimental setup used for trapping PS particles. 100x objective with oil is used for focusing the laser onto the gold substrate. $532 \mathrm{~nm}$ green laser is used to incite resonance heating in AuNI substrate. Low concentration of PS particles is dispersed in CTAC solution to avoid inter-particle interactions and sonicated for 5 minutes. The trapped particle is tracked using Andor Fast Camera (1000 fps). A gaussian beam is fitted to the X trajectory of the 
trapped particle and the standard deviation of the fit is used to determine the trapping stiffness of the particle as

$$
\kappa=\frac{k_{B} T}{\sigma^{2}}
$$

where $\kappa$ is the trapping stiffness and $\sigma$ is the standard deviation of the Gaussian fit for histogram of $\mathrm{X}$ coordinates. Figure $\mathrm{S} 4$ shows a sample of $\mathrm{X}$ trajectory of $1 \mu \mathrm{m}$ PS particle in CTAC of $1 \mathrm{mM}$ solution and the corresponding Gaussian fit.
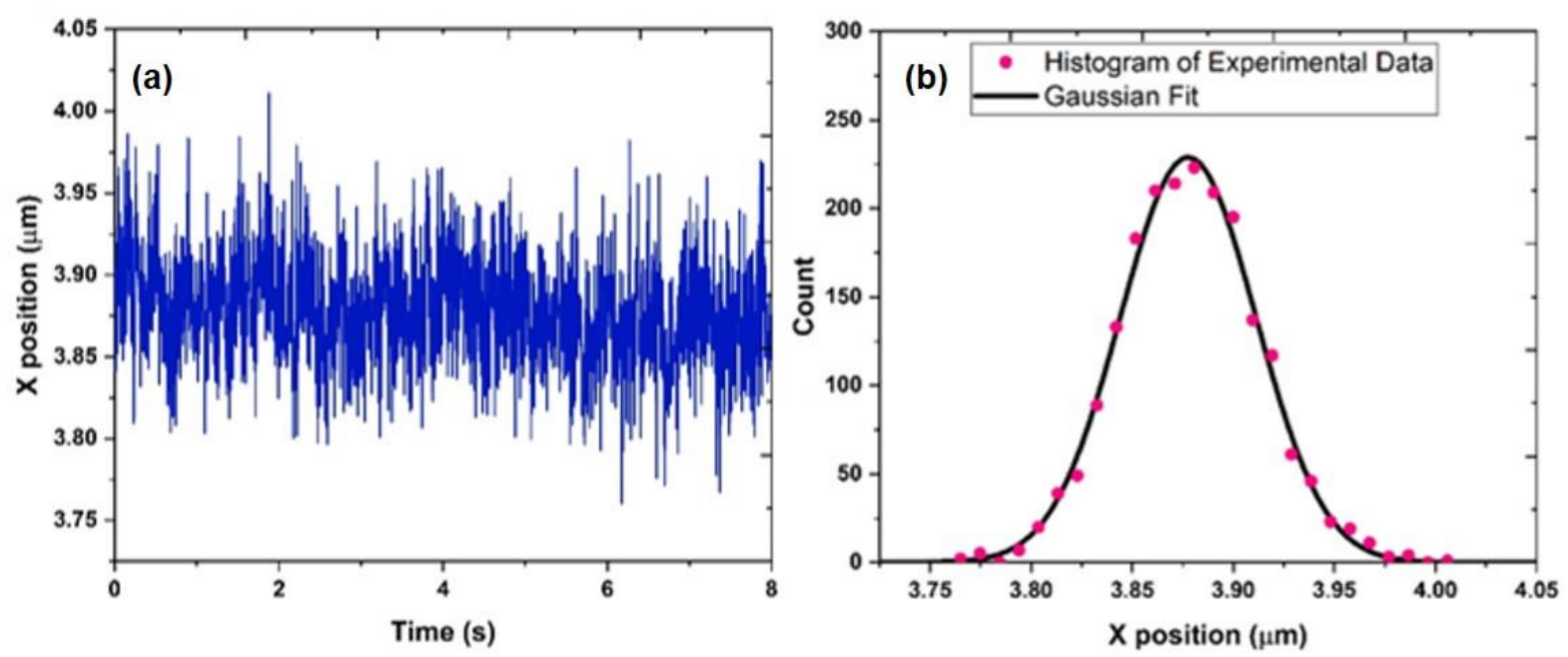

Figure S4: (a) X trajectory of trapped particle obtained using fast camera. (b) Histogram of the trajectory is obtained, and a Gaussian is fitted to the data to evaluate trapping stiffness of the particle. 


\section{Supplementary Note V - Optical force and osmotic force on the particle}

Optical forces are evaluated on the particle using commercially a vailable software, Lumerical FDTD simulations (FDTD solutions, Lumerical Inc). The refractive index of polystyrene particles as a function of wavelength is taken from the work of Sultanova et. al. ${ }^{4}$. Figure S5 gives a measure of the net optical force on the particle as a function of the distance from the beam. Beam size is assumed as $0.8 \mu \mathrm{m}$ from intensity profile measured using a charge-couple device (CCD).

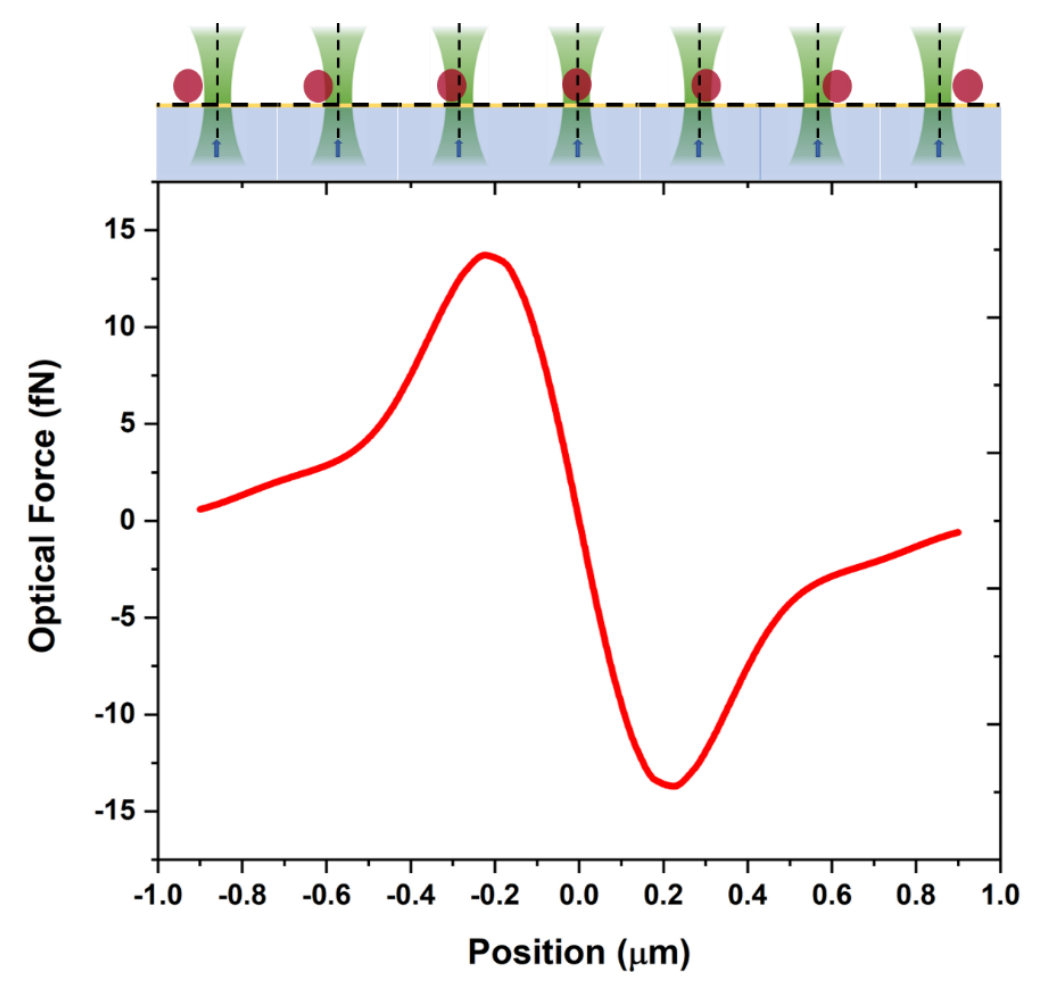

Figure S5: In-plane optical force on the particle with beam center as position reference

The maximum optical force on the particle is about $15 \mathrm{fN}$. The forces quickly attenuate with the particle's distance from the center of the beam. Compared to long range trapping and $\mathrm{pN}$ scale thermo-electric forces, the optical forces are 2-3 orders lower in magnitude and therefore can be neglected. Osmotic force on the particle is evaluated using numerical integration of osmotic pressure on the particle. The osmotic pressure on the particle varies over its surface due to the change in 
salinity resulting from the temperature gradient during heating of the substrate. The salinity is solved using finite element analysis software (COMSOL Multiphysics® v. 5.3., www.comsol.com) by solving the mass flux equations with constant concentration boundary conditions using small gradient approximations.

$$
\begin{gathered}
J_{C l}=0=-D_{C l} \nabla c_{C l}-c_{C l} D_{T_{C l}} \nabla T \\
J_{m i c}=0=-D_{m i c} \nabla c_{m i c}-c_{m i c} D_{T_{m i c}} \nabla T \\
c_{C l} \rightarrow n_{0} \text { at } x \rightarrow \infty \\
c_{m i c} \rightarrow \frac{\left(n_{0}-n_{c m c}\right)}{N_{a g g}} \text { at } x \rightarrow \infty
\end{gathered}
$$

where $J$ represents the ions flux assuming no effect of thermoelectric field on the concentration profile (small-gradient approximation), $D$ is the diffusion coefficient of the ion, $c$ is the concentration of ion, $D_{T}$ is the thermo-diffusion coefficient of the ion. Subscripts $C l$ and mic represents Chloride ion and CTAC micelles respectively.

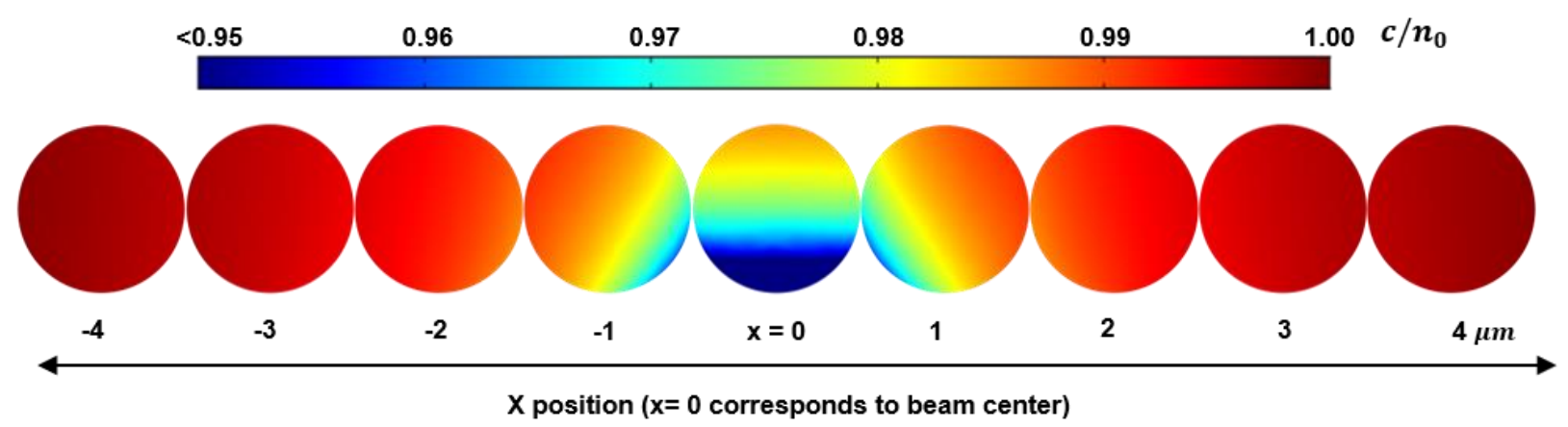

Figure S6: Ratio of salinity of ion around the particle surface and concentration of surfactant in free solution at constant temperature obtained at a laser power of $0.50 \mathrm{~mW}$. The color gradient on the particle gives an estimate of the osmotic force direction acting on the particle, and the resultant $\mathrm{X}$ component of the force is used for trapping stiffness evaluation. 
Figure S6 represents the resultant concentration of the ions/surfactant on the particle. The concentration of ions is numerically integrated to evaluate osmotic force on the particle as ${ }^{5}$

$$
\begin{gathered}
\Pi=N_{A} c_{C l} k_{B} T+N_{A} c_{m i c} k_{B} T\left(1+\alpha+\alpha^{2}-\alpha^{3}\right)\left(1-\alpha^{3}\right)^{-3} \\
F_{\text {osmotic }}=\int \Pi \sin (\theta) d \theta d \phi=\sum_{\theta=0}^{\pi} \sum_{\phi=0}^{2 \pi} \Pi \sin (\theta) d \theta d \phi
\end{gathered}
$$

Here $\alpha$ is the correction factor to account for the size of the micelles $(\sim 3 \mathrm{~nm})$ and is the effective micelle volume fraction given as

$$
\alpha=\frac{4 \rho}{3} \pi L_{e f f}^{3} ; L_{e f f}=r_{m i c}+2 \kappa^{-1} ; \rho=N_{A} / N_{a g g}\left(n_{0}-n_{C M C}\right)
$$

where $r_{\text {mic }}$ is the radius of the micelle, $\kappa^{-1}$ is the inverse Debye length obtained as a function of concentration. The resultant force on the particle is of the order of $\mathrm{fN}$ and therefore osmotic force is also neglected in determination of trapping stiffness. 


\section{Supplementary Note VI - Comprehensive details of simulation}

The incident laser power is measure d and absorption coefficient of the gold NI is taken as $0.2^{6}$. The beam size is obtained as $0.75 \mu \mathrm{m}$ by Gaussian fitting of the intensity profile obtained using CCD. Initially an intensity $\left(Q_{\max }\right)$ is assumed and a Gaussian heat influx with beam diameter $=0.75 \mu m$ is taken as the interface towards the water medium. The net heat influx into water medium is taken as

$$
P_{\text {water }}=\frac{Q_{\max } w_{0}^{2}}{2}
$$

The water medium is solved for, with constant temperature boundary conditions and the temperature at the interface is obtained. This temperature at the interface is assumed as the temperature source towards the glass substrate and the net heat power into glass is obtained from the finite element analysis software (COMSOL Multiphysics ${ }^{\circledR}$ v. 5.3., www.comsol.com). The total power towards water medium and glass is obtained is correlated to the measured power. Inverting the linear equation, a relation between incident power and assumed intensity of the Gaussian heat influx is obtained.

$$
P_{\text {laser }}=\frac{P_{\text {absorbed }}}{\alpha}=\frac{P_{\text {water }}+P_{\text {glass }}}{\alpha}=\frac{\frac{1}{2} Q_{\text {max }} w_{0}^{2}+P_{\text {glass }}}{\alpha}
$$

Once the relation between $Q_{\max }$ and $P_{\text {laser }}$ is obtained, we can solve the temperature distribution of the computational geometry and export the data around the PS particle (Figure S7). Thus, a relation between the assumed power and incident laser, and the temperature distribution is obtained. The temperature and its gradient around the particle are retrieved and resolved into parallel (tangential) and perpendicular (normal) components in MATLAB (version 2018b, Natick, Massachusetts, The Mathworks Inc.) and thermoelectric field formulation is applied to determine the thermoelectric force on the particle at a particle position. This is repeated for varying simulations of particle positions along $\mathrm{X}$ direction and thermoelectric force is obtained a function of particle position. 
(a)

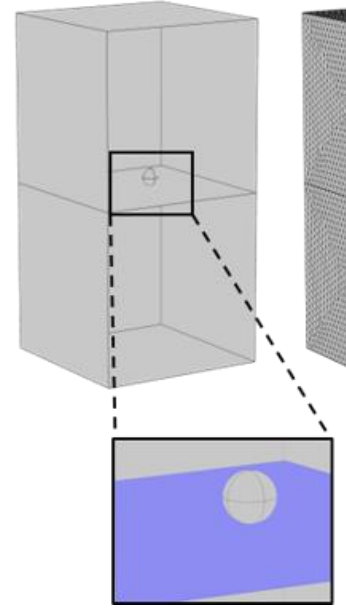

(b)

(c)

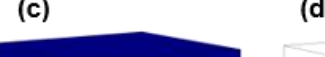

(d)

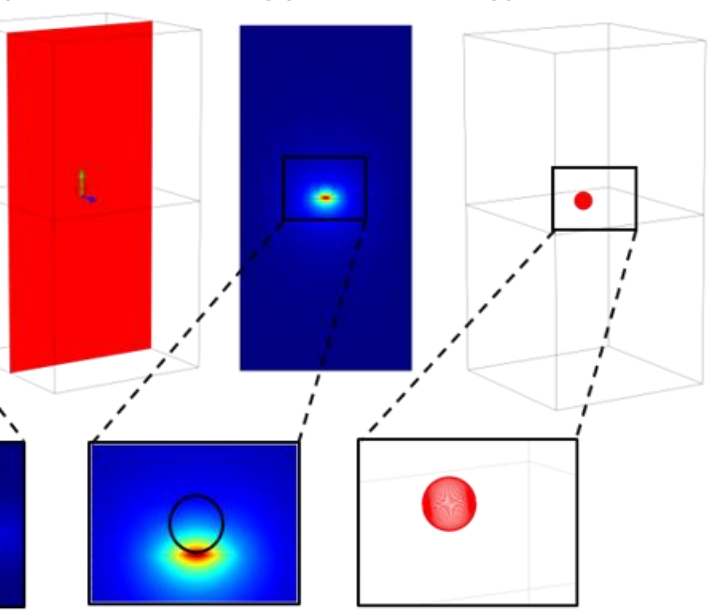

Figure S7: (a) Schematic of the simulation, upper cube is water, lower cube is glass, sphere represents PS particle. The blue surface in the zoomed portion is the interface where Gaussian heating is given as an input (b) Mesh of the schematic (c) Temperature profile evolved after laser beam is modelled as Gaussian heat flux (d) XZ plane on which the particle is moved along $\mathrm{X}$ direction (e) Temperature of the system on $\mathrm{XZ}$ plane shows that temperature is almost continuous at the water-PS surface but the temperature gradient reveals that it is not perfectly continuous (f) The points on the PS sphere where data is collected and exported for post-processing. 


\section{Supplementary Note VII - Comparison of the rmoelectric forces using a. Nume rical integration (this work) and b. Known corrective term for the rmal conductivity contrast}

For a laser power of $135 \mu \mathrm{W}, \mathrm{CTAC}$ concentration of $1 \mathrm{mM}$ and a PS particle size of $1 \mu \mathrm{m}$, the thermoelectric force is estimated to be $0.2967 \mathrm{pN}$ based on this work. We compare this with the thermoelectric force obtained by treating the particle as a point-particle $\left(T E_{\mathrm{Force}, \mathrm{C}}\right)$ and is evaluated as

$$
T E_{\mathrm{Force}, \mathrm{C}}=q \xi S C \nabla T
$$

where $q$ is the charge on the particle (obtained at a zeta potential of $59 \mathrm{mV}$ ), $S C$ is the Seebeck Coefficient and $\nabla T$ is the temperature gradient at the point-particle. $\xi$ is the correction term that

includes the thermal conductivity contrast as $\frac{3 k_{\mathrm{S}}}{2 k_{\mathrm{S}}+k_{\mathrm{P}}}$ where $k_{\mathrm{S}}$ is the thermal conductivity of the solvent and $k_{\mathrm{P}}$ is the thermal conductivity of the particle. Thermal conductivity of PS particles is assumed as $0.15 \mathrm{~W} / \mathrm{m} \cdot \mathrm{K}^{7}$ and thermal conductivity of water is $0.59 \mathrm{~W} / \mathrm{m} . \mathrm{K}$ at room temperature. The $T E_{\text {Force,C }}$ obtained is $0.3427 \mathrm{pN}$ and a good agreement is observed between these two forces, thereby working as a proof of concept. Although the corrective term is limited to spherical is otropic particles, the current methodology can be extended to any particle of known thermal conductivity contrast within the particle and is also applicable to non-spherical particles. 


\section{References:}

(1) Fayolle, S.; Bickel, T.; Le Boiteux, S.; Würger, A. Thermodiffusion of Charged Micelles. Phys. Rev. Lett. 2005, 95 (20), 1-4. https://doi.org/10.1103/Phys RevLett.95.208301.

(2) Vigolo, D.; Buzzaccaro, S.; Piazza, R. Thermophoresis and Thermoelectricity in Surfactant Solutions. Langmuir 2010, 26 (11), 7792-7801. https://doi.org/10.1021/la904588s.

(3) Roelants, E.; De Schryver, F. C. Parameters Affecting Aqueous Micelles of CTAC, TTAC, and DTAC Probed by Fluorescence Quenching. Langmuir 1987, 3 (2), 209-214. https://doi.org/10.1021/la00074a011.

(4) Sultanova, N. G.; Nikolov, I. D.; Ivanov, C. D. Measuring the Refractometric Characteristics of Optical Plastics. Opt. Quantum Electron. 2003, 35 (1), 21-34. https//doi.org/10.1023/A:1021811200953.

(5) Iracki, T. D.; Beltran-villegas, D. J.; Eichmann, S. L.; Bevan, M. A. Charged Micelle Depletion Attraction and Interfacial Colloidal Phase Behavior. 2010, 26 (25), 1871018717. https://doi.org/10.1021/la103701k.

(6) Gupta, G.; Tanaka, D.; Ito, Y.; Shibata, D.; Shimojo, M.; Furuya, K.; Mitsui, K.; Kajikawa, K. Absorption Spectroscopy of Gold Nanoisland Films: Optical and Structural Characterization. Nanotechnology 2009, 20 (2), 1-9. https://doi.org/10.1088/09574484/20/2/025703.

(7) Agarwal, S.; Patidar, D.; Saxena, N. S. Effective Thermal Conductivity of CdS/ZnS Nanoparticles Embedded Polystyrene Nanocomposites. Heat Mass Transf. und Stoffuebertragung 2013, 49 (7), 947-953. https://doi.org/10.1007/s00231-013-1138-7. 